\title{
Propionic acid intervention in obese Ldlr-I-.Leiden mice attenuates NASH development, but negatively affects cognition
}

\author{
$\underline{\text { Eveline Gart }^{1}}$, Anouk Tengeler ${ }^{2}$, Martine Morrison ${ }^{1}$, Tamas Kosicz ${ }^{3}$, Wim van Duyvenvoorde ${ }^{1}$, \\ Robert Kleemann ${ }^{1}$ and Amanda Kiliaan ${ }^{2}$ \\ ${ }^{1}$ TNO, Leiden, Netherlands, \\ ${ }^{2}$ Radboud university medical center, Nijmegen, Netherlands and \\ ${ }^{3}$ Mayo Clinic, Rochester, USA
}

\begin{abstract}
Introduction: There is an increasing interest to elucidate the health effects of short-chain fatty acids (SCFAs) on metabolism, obesity and brain function. Obesity is often associated with the development of non-alcoholic steatohepatitis (NASH) and cognitive impairment. We herein investigated potential health effects of the SCFA propionic acid (PA) on NASH development and brain function including cognition and behavior readouts.
\end{abstract}

Materials and Methods: During 17 weeks of run-in, LDLR-/-.Leiden mice received either high-fat diet (HFD) to establish obesity or chow as control. Obese mice were matched into groups $(n=15 /$ group $)$ and treated with propionic acid $(\mathrm{PA}+\mathrm{HFD})$, or a reference fatty acid (caproic acid; CA + HFD), or HFD without supplements (HFD). Cognitive and behavioral effects, as well as metabolic and inflammatory risk factors, were assessed prior to and after 12 weeks of treatment. At endpoint, liver and adipose tissue pathology were histologically and biochemically analyzed.

Results: PA, but not reference CA, reduced body weight and this effect was independent of food intake. PA also reduced fasting insulin levels and plasma cholesterol levels relative to the start of intervention. In addition, PA reduced total and subcutaneous fat mass, but did not affect WAT inflammation. Histopathological analysis of the liver demonstrated that PA reduced macrovesicular steatosis, hypertrophy and inflammation. Consistent herewith, PA reduced the inflammatory marker serum amyloid A and tended to increase intrahepatic ketone bodies ( $\beta$-hydroxybutyrate), and lowered the hepatic collagen content. PA treatment did not affect normal behavior in the open field test but mice showed impaired spatial memory, i.e. the latency to find the platform in the Morris water maze was increased. In line with these findings, synaptophysin expression in the hippocampus and vasoactivity in both the cortex and hippocampus and mitochondrial activity in the cerebellum was decreased by PA. The reference fatty acid CA exerted no effects on the above readouts.

Discussion: Overall, PA exerted pronounced positive effects on metabolic risk factors (insulin, cholesterol, adipose mass) and attenuated the development of NASH and liver fibrosis. The observed higher levels of intrahepatic ketone bodies suggest that lipid $\beta$-oxidation is increased which may contribute to the health effects in the liver and reduced fat mass. Contrarily, PA negatively affected spatial memory, which could be a result of reduced synaptophysin expression and decreased mitochondrial function in the brain leading to impaired neurotransmitter signaling and cognitive performance.

\section{Conflict of Interest}

There is no conflict of interest 